\title{
A novel energy-motion model for continuous sEMG decoding: from muscle energy to motor pattern
}

\author{
Gang Liu, Lu Wang and Jing Wang
}

scoop before peer-review

\begin{abstract}
Background: At present, the gesture recognition using sEMG signals requires vast amounts of training data or limits to a few hand movements. This paper presents a novel dynamic energy model that can decode continuous hand actions with force information, by training small amounts of sEMG data.

Method: As activating the forearm muscles, the corresponding fingers are moving or tend to move (namely exerting force). The moving fingers store kinetic energy, and the fingers with moving trends store potential energy. The kinetic and potential energy of fingers is dynamically allocated due to the adaptive-coupling mechanism of five-fingers in actual motion. At this certain moment, the sum of the two energies is constant. We regarded energy mode with the same direction of acceleration of each finger, but likely different movements, as the same one, and divided hand movements into ten energy modes. Independent component analysis and machine learning methods were used to model associations between sEMG signals and energy mode, to determine the hand action, including speed and force adaptively. This theory imitates the self-adapting mechanism in the actual task; thus, ten healthy subjects were recruited, and three experiments mimicking activities of daily living were designed to evaluate the interface: (1) decoding untrained configurations, (2) decoding the amount of single-finger energy, and (3) real-time control.

Results:(1) Participants completed the untrained hand movements $(100 / 100, \mathrm{p}<0.0001)$. (2)

The test of pricking balloon with a needle tip was designed with significantly better than chance $(779 / 1000, \mathrm{p}<0.0001) .(3)$ The test of punching a hole in the plasticine on the balloon was with over $95 \%$ success rate $(97.67 \pm 5.04 \%, \mathrm{p}<0.01)$.

Conclusion: The model can achieve continuous hand actions with force information, by training small amounts of sEMG data, which reduces trained complexity.
\end{abstract}

Keywords: myoelectric interface, amputees, prosthetic hand, electromyogram, real-time systems, conservation of energy.

\section{Introduction}

Hand loss is a highly disabling event, and markedly affects the quality of life [3]. In order to replace the capabilities lost, the replacement should be designed to faithfully mimic the native hands, providing the user with intuitive control, sufficient feedback, and multiple functions (Fig. 1) [3-6]. Sixty years ago, the advent of a myoelectric prosthetic hand, an externally powered prosthesis extracting motion intent from electromyogram (EMG) signals, brought a promising approach [7]. However, myoelectric prosthetic hands with intuitive control and multiple functions have not been widely utilized in the commercial area.

The first or earliest type only has two motions, e.g., hand open or close [8-10]. Two bipolar EMG electrodes are placed at the flexors and extensors of the residual limb to record information about the neuromuscular activity. The second type is based on motion classification that assigns EMG features to a discrete set of motions [11-13]. Tremendous success has been achieved in this line of research. Recently, Furui et al. have demonstrated that a mechanism classifying unlearned combined motions from a dataset of learned 
motions [13]. In principle, it can be extended to the classification of any hand motions. Despite its success in research, classification in its basic form only allows for the sequential activation of motions, precluding intuitive control of smooth movements. An error may lead to an utterly unwanted action that may compromise the full task [5].

Recently, to overcome the limitations of the classification-based approaches, regression-based approaches have been proposed. Most research has focused on the estimation of kinematic joint angles of the wrist [1416]. Recently, Zhuang et al. succeed in the estimation of the force of the single finger [17]. However, this technique requires considerable training and experience due to the mechanical coupling and physiological coupling of fivefingers $[1,2,11,18,19]$.

Here, this paper proposes a myoelectric interface based on the conservation of energy (Fig. 2 and Fig. 3). For an instant muscular activation associated with hand movements is constant, the five-fingers are in a state of motion, tending to move or relaxation; In the view of energy, at some point in time of muscular activation, the energy of the whole hand is a constant. However, the form of five-fingers may be kinetic energy, potential energy, or non-energy. If the five-fingers state is in the same direction of acceleration, we regard it as the same energy mode (Fig. 1). Therefore, one energy mode can achieve multiple motor patterns. In this way, we can map massive motor patterns from sEMG by small sample training by this approach.

This paper consists of the proposed myoelectric interface, three sets of exploratory operational experiments, and offline analysis. Section II introduces an overview of the bionic interface. Section III describes the details of the interface and experiments. Section IV shows the results of experiments and off-line analysis. In Section V, we discuss the results and limitations and conclude. Note that we provided Supplementary Materials and Movies, and "Fig. S" can be found in Supplementary Materials.

\section{Bionic inspiration and interface overview}

Inspired by the neuromuscular system of the human hand, we propose a myoelectric interface based on energy allocation. The scheme for the strategy is shown in Fig. 2. We imitate two stages that the central nervous system (CNS) activates muscles and that muscles control fingers to complete the task adaptively (Fig. 2a). Strong evidence from EMG of frogs [20], cats [21], primates [22], and humans [23] has demonstrated the existence of muscle synergy in the neuromuscular system. For the first stage, a group of synergetic muscles instead of one is activated to perform a certain task, which allows the CNS to achieve muscle activation by controlling a few synergies. For the second stage, the energy of muscles is transferred through mechanical coupling to five-fingers, which performs manual

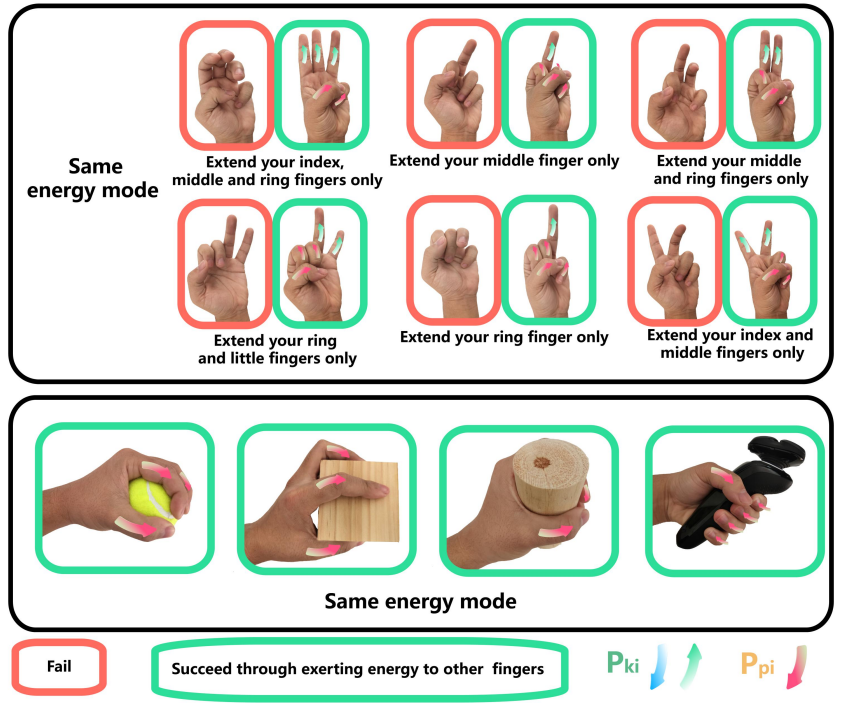

Fig. 1. Example of the primary mechanism of human hand mimicked in the present study. Active movement at one finger may result in some movement at other fingers unless activating corresponding muscles to keep other fingers static. Humans rarely move one finger alone, but multiple fingers simultaneously, and the energy adaptively extend to multiple motions or forces according to the task itself $[1,2]$. We sought to regard the whole manual task as energy transfer, mimicking the adaptive mechanism of the human hand. The direction of arrows indicates the direction of acceleration. If the five-fingers state is in the same direction of acceleration, we regard it as the same energy mode. $P_{k i}$ expresses the kinetic energy of the $i$-th finger, while $P_{p i}$ expresses the potential energy of the $i$-th finger.

tasks by the conversion of kinetic energy and potential energy adaptively $[2,24]$.

In light of this, we modularize the model of the myoelectric interface (Fig. 2b). Firstly, control signals are obtained using the filtered EMG recordings as input to a synergy matrix that represents muscle activation strategies from the individual muscles to muscle groups, which highlight the information of synergies from CNS. Secondly, controlling fingers through the muscles are regarded as an energy transfer process based on energy conservation.

To unify the static force and motion condition, according to Newton's Second Law, we assume that a fictitious resistance exists in fingertip during flexion and extension of each finger, and muscle activation aims to overcome the resistance. Thus, according to energy conservation, the total energy of a finger can be regarded as a sum of the kinetic and potential energy, consisted of three forms: kinetic energy, potential energy, or coexistence of kinetic energy and potential energy. At a certain moment of muscle activation, the energy allocation of five fingers is determined accordingly. For a single finger, the total energy (kinetic energy plus potential energy) is also determined. Resistance catalyzes the interconversion between kinetic energy and potential energy, but the total amount is constant.

The essence of the myoelectric interface is the total energy allocation of five-fingers. Therefore, we can solve this problem through two extreme conditions of energy transfer. 


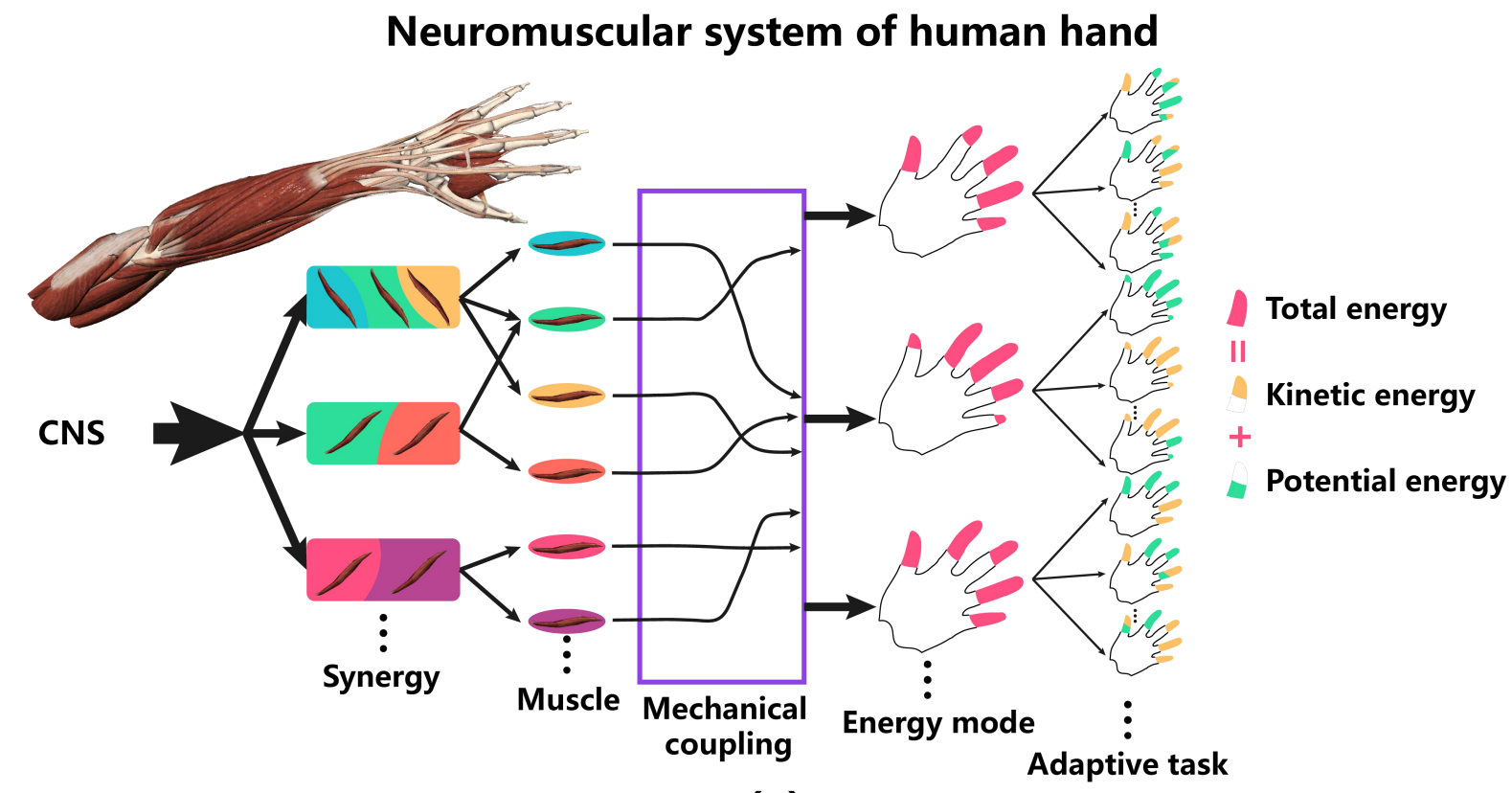

(a)

\section{Myoelectric interface based on energy allocation}

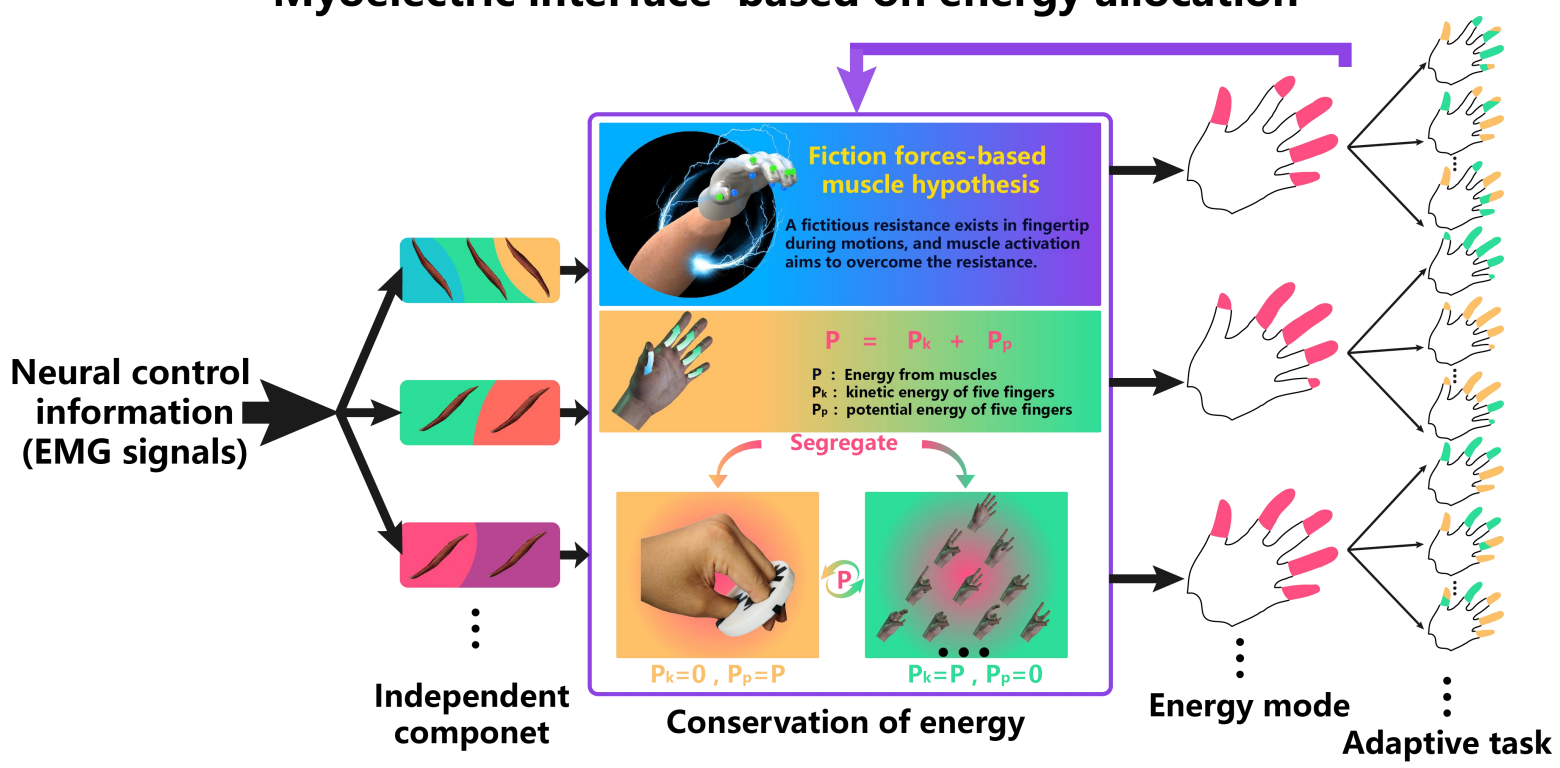

(b)

Fig. 2. Scheme for the biomimetic myoelectric interface. (a) Schematic diagram of the neuromuscular system of human hand, including muscle synergy, mechanical coupling, and the conversion of kinetic energy and potential energy adaptively. (b) Schematic diagram of the proposed myoelectric interface. The energy of muscles is transferred through mechanical coupling to five-fingers, which performs manual tasks by the conversion of kinetic energy and potential energy adaptively. The essence of the myoelectric interface is the total energy allocation of five-fingers. Furthermore, the size of colored area on single finger expresses the amount of energy. The aim is to solve for the pink area of five-fingers (allocation of total energy).

Figure 3 shows an overview of the proposed myoelectric interface. To simplify the model, we utilize the conditions of wholly transferred kinetic energy or potential energy. The five-fingers energy of the operator was estimated by the bionic model using independent component analysis (ICA) and conservation of energy, thereby allowing the expression of the unlearned hand tasks via a few learned energy modes adaptively $[2,25]$.

\section{Materials and methods}

\subsection{Scheme for the bionic myoelectric interface}

From the perspective of information and energy transfer, the neuromuscular system of humans hand consists of muscle synergy, mechanical coupling, and the adaptive conversion of kinetic energy and potential energy (Fig. 2 and 

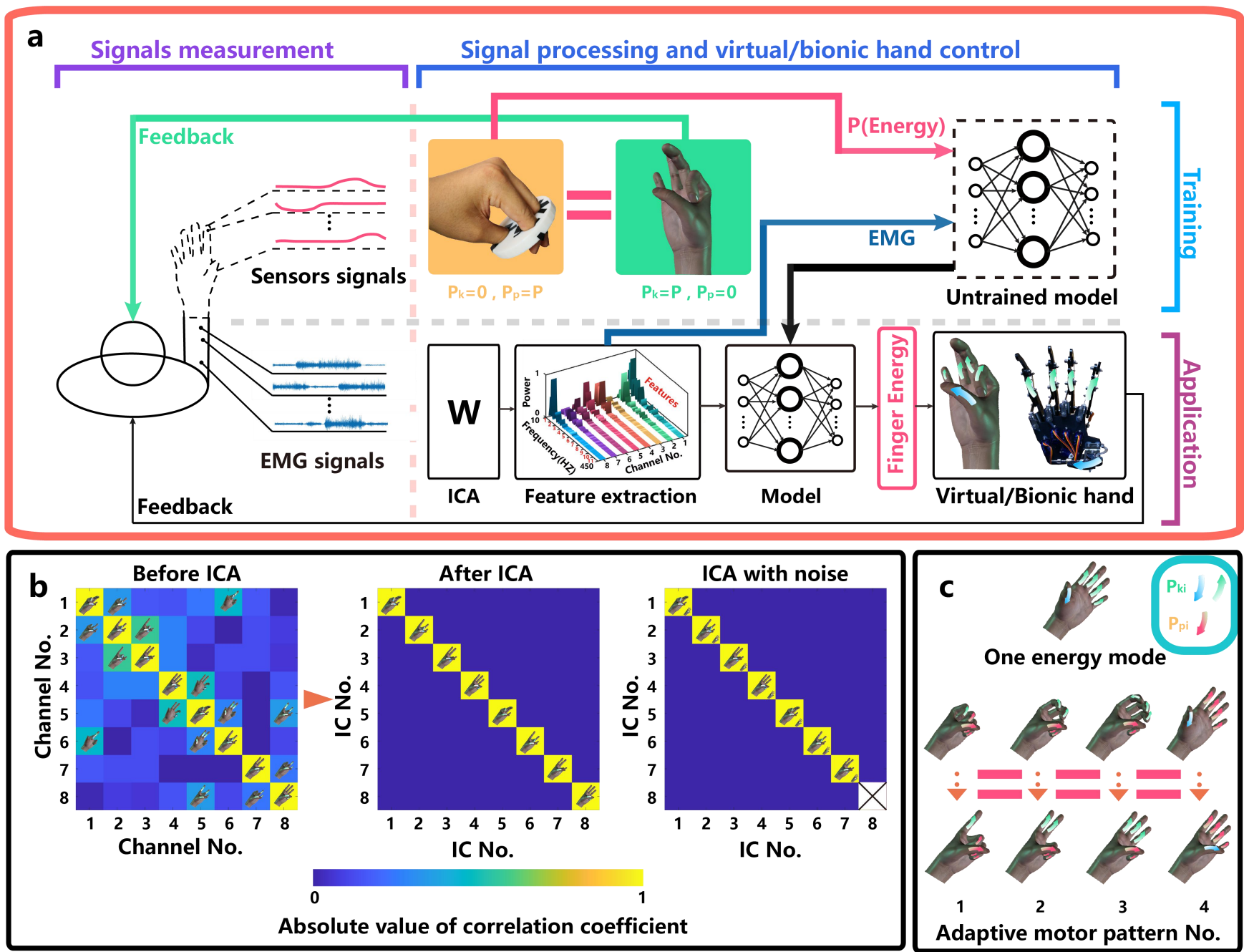

Absolute value of correlation coefficient

Adaptive motor pattern No.

Fig. 3. Overview of the myoelectric interface based on the conservation of energy. The myoelectric interface (a) is composed of training and application stages. Both stages consist of signals measurement and signal processing and virtual/bionic hand control. Note that, in the training stage, the sensor signals can be measured on the intact side of the user. Besides, independent component analysis (ICA) decomposes a synergy matrix that represents muscle activation. The synergy matrix is used to extract muscle synergy and improve robustness (b). Furthermore, each energy mode can extend many other hand tasks adaptively (c, an example, the direction of the arrow represents the direction of acceleration of single finger, and all adaptive motor patterns are the same energy mode.). $P_{k i}$ expresses the kinetic energy of the $i$-th finger, while $P_{p i}$ expresses the potential energy of the $i$-th finger.

Fig. 1). Therefore, we mimic these parts to designed our model.

\subsubsection{ICA to mimic muscle synergy.}

Evidence from a large number of animal experiments has been demonstrated that the CNS achieves muscle activation by controlling a small number of synergies rather than controlling individual muscles [20-22, 26, 27]. Briefly, muscle synergy pattern can be expressed by the activation of individual muscles $\left[m_{1}(t), m_{2}(t), \cdots, m_{n}(t)\right] \in \mathfrak{R}^{n}$ ( $n$ is the number of muscle)

$$
u(t)=F^{\text {trans }}\left(m_{1}(t), m_{2}(t), \cdots ; m_{n}(t)\right)
$$

Where $u(t)=\left[u_{1}(t), u_{2}(t), \cdots, u_{m}(t)\right] \in \mathfrak{R}^{m} \quad(m$ is the number of synergy patterns and $m \leq n)$ and $F^{\text {trans }}(\cdots)$ is a function that transforms the muscle activation into a smaller number of synergies. Previous experiments of frogs [26] and rats [27] demonstrated that we could use ICA to extract these muscle synergy patterns. Therefore, muscle synergy pattern $u(t)$ is extracted from the time-series EMG pattern $\left[s_{1}(t), s_{2}(t), \cdots, s_{n}(t)\right] \in \Re^{c} \quad(c \quad$ is the number of EMG electrodes and $c \geq m$ )

$$
u(t)+\mathrm{e}(t)=F^{\text {ICAtrans }}\left(s_{1}(t), s_{2}(t), \cdots s_{c}(t)\right)
$$

Where $F^{I C A t r a n s}(\cdots)$ is a function that transforms the timeseries EMG pattern into a smaller number of synergies, and $e(t)$ is the noise of the system (Fig. 3b). $u(t)$ is regarded as the information from CNS.

\subsubsection{Energy transfer applied to the human hand.}

Both mechanical coupling and neuromuscular control limit finger independence $[1,2]$. For example, active movement at one finger may lead to some movement at another finger (Fig. 1). To implement the fluid decoding of a single finger, we, 
therefore, sought to regard the whole manual task as energy transfer, mimicking the adaptive mechanism of the human hand. Applying the conservation of energy to the human hand can be expressed as the following formula (Fig. 2b).

$$
\begin{aligned}
& P=P_{K}+P_{P} \\
& \Delta P_{K}=-\Delta P_{P}
\end{aligned}
$$

Where $P$ is the energy from muscles to hand, $P_{K}$ is the kinetic energy of hand, $P_{P}$ is the potential energy of hand (usually existing in the form of strain energy), $\Delta P_{K}$ is the amount of change of kinetic energy, and $\Delta P_{P}$ is the amount of change of potential energy. Also, the energy transfer can be phrased as follows at the level of a single finger.

$$
\begin{gathered}
P=\sum_{f=1}^{5} P_{f}^{s}, \quad P_{K}=\sum_{f=1}^{5} P_{k, f}, \quad P_{P}=\sum_{f=1}^{5} P_{p, f} \\
\Delta P_{P}=\sum_{f=1}^{5} \Delta P_{p, f}, \quad \Delta P_{K}=\sum_{f=1}^{5} \Delta P_{k, f}
\end{gathered}
$$

Where $\left\{P_{1}^{s}, P_{2}^{s}, \cdots, P_{5}^{s}\right\} \in \mathfrak{R}^{5},\left\{P_{k, 1}, P_{k, 2}, \cdots, P_{k, 5}\right\} \in \mathfrak{R}^{5}$ and $\left\{P_{p, 1}, P_{p, 2}, \cdots, P_{p, 5}\right\} \in \Re^{5}$ are the total, kinetic, potential energy for each finger, respectively. $\left\{\Delta P_{k, 1}, \Delta P_{k, 2}, \cdots, \Delta P_{k, 5}\right\} \in \Re^{5}$ and $\left\{\Delta P_{p, 1}, \Delta P_{p, 2}, \cdots ; \Delta P_{p, 5}\right\} \in \mathfrak{R}^{5}$ are the amount of change of kinetic, potential energy for each finger, respectively. Uppercase subscript of "P" or "K" expresses the whole hand level. Lowercase subscript of " $p$ " or " $k$ " expresses the single finger level. Superscript "s" indicates the single finger level. In addition, a single finger satisfies the following conditions.

$$
P_{f}^{s}=P_{k, f}+P_{p, f}, \quad f \in\{1,2,3,4,5\}
$$

Here, according to the adaptive mechanism of the fingers in task [1,2] and muscle synergy [13], we assume that a fictitious resistance exists in fingertip during flexion and extension of each finger, and muscle activation aims to overcome the resistance, so as to unify the static force and motion condition (Newton's Second Law of Motion-Force and Accelerat). Thus, the energy transfer of the single finger is as follows.

$$
\Delta P_{k, f}=-\Delta P_{p, f}, \quad f \in\{1,2,3,4,5\}
$$

In other words, for a certain muscle activation, although the form of energy is uncertain, the total energy of the single finger is a constant. Therefore, for the five-fingers level, we do not care the form of energy, and this process recasts the decoding problem as the problem of energy allocation of the five-fingers (energy mode; the adaptive and expansive expression are shown in Fig.3c).

To simplify the model, we utilize the conditions of wholly transferred external kinetic energy $P_{K}^{e x t}$ or external potential energy $P_{P}^{e x t}$.

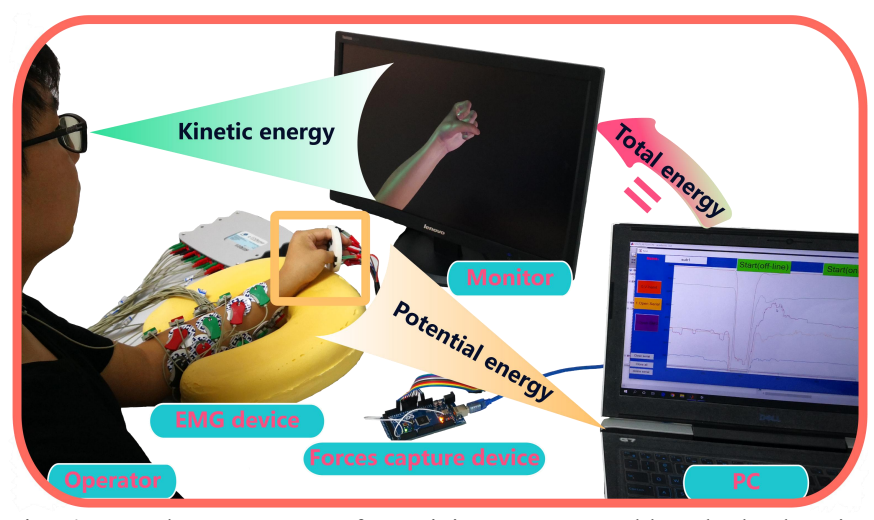

Fig. 4. Hardware structure for training states. Although the learning method we selected, such as ANN or SVM, is similar to previous researches that recorded motions or force of the wrist, the difference of our interface is that we had users perform tasks that represent energy modes in the level of the whole hand. We found it is very convenient to extend massive manual tasks by training a few specific energy modes based on virtual hand visualization about the entire hand, rather than a lot of motions or singlefinger force. Notice the gesture in yellow box is without mechanical coupling, so we record the wholly transferred potential energy. ( Ten energy modes in Movie S6.)

$$
P_{K}^{e x t}=P_{P}^{e x t}=P
$$

Also, the form of a single finger is as follows.

$$
P_{k, f}^{e x t}=P_{p, f}^{e x t}=P_{f}^{s}, \quad f \in\{1,2,3,4,5\}
$$

Where $\quad P_{k, f}^{\text {ext }} \in\left\{P_{k, 1}^{\text {ext }}, P_{k, 2}^{\text {ext }}, \cdots, P_{k, 5}^{\text {ext }}\right\} \in \mathfrak{R}^{5} \quad$ and $\quad P_{p, f}^{\text {ext }} \in$ $\left\{P_{p, 1}^{\text {ext }}, P_{p, 2}^{\text {ext }}, \cdots, P_{p, 5}^{\text {ext }}\right\} \in \mathfrak{R}^{5}$ are the wholly transferred kinetic energy or potential energy for a single finger. Furthermore, with the help of an additional system, according to the principle of virtual work, the wholly transferred potential energy should be equal to the amount of change of internal energy of the additional system (Fig. 2b and Fig. 3a).

$$
P_{p, f}^{e x t}=\mathrm{W}_{p, f}^{e x t}=F_{f}^{e x t} \cdot \delta, \quad f \in\{1,2,3,4,5\}
$$

Where $W_{p, f}^{e x t}$ is the work of the energy transfer from finger to the additional system, $F_{f}^{e x t}$ is the force applied to the additional system, and $\delta$ is the virtual displacement. Besides, (8) compared with (1), the energy of the five-fingers is expressed as the following formula.

$$
P_{f}^{s}=P_{k, f}^{e x t}=P_{p, f}^{e x t}=F_{f}^{e x t} \cdot \delta, \quad f \in\{1,2,3,4,5\}
$$

Then, we utilize the two extreme conditions of energy transfer simultaneously to solve the total energy for each finger, as shown in figure $3 \mathrm{a}$.

\subsection{Experimental protocol}


We performed off-line analysis and three sets of operational experiments in which we decoded the finger energy of subjects using EMG signals recorded from their forearms. We recruited ten able-bodied subjects (subjects 1, $2,3, \ldots, 10$ ) for the study (note that the EMG signals from subject 10 are contaminated with noise due to electrode shifts [28] and one lift-off electrode [29] intentionally in training stage).

\subsubsection{Subjects and EMG recording.}

In this study, 10 able-bodied subjects (two females, eight males, aged $26.4 \pm 1.43$ years) gave informed consent to participate in the experiment protocol. All participants were right-handed. We performed training stages in all experiments with their left hands for convenient operations. For the right hands, the electrodes were placed in the same positions as the left hand in operational experiments. Also, these subjects' hands are similar in size due to the fixed device or forces capture device (Fig. 4).

According to anatomy and kinesiology of hand [30], we recorded the EMG activity from eight extrinsic muscles of the forearm of all subjects. As shown in figure S3, four flexor muscles and four extensor muscles related to hand or finger movements were selected.

\subsubsection{Training stages.}

\section{a. System setup}

The system is mainly composed of a multichannel surface electromyography device, a fixed device or force capture device, and a personal computer (Fig. 4). Surface EMG signals, as well as the continuous five-fingers forces in both finger flexion and extension directions with visual feedback of virtual hand, were recorded simultaneously. Among them, proportional five-fingers forces indicate the potential energy that converted into the internal energy of the capture device. Visual feedback of the virtual hand represents kinetic energy that is completely converted by potential energy for each finger (kinetic energy is in direct proportion to the square of the speed of virtual fingers). Formula derivation is as follows.

For potential energy of five-fingers:

$$
\begin{aligned}
& P_{p, 1}^{e x t}: P_{p, 2}^{e x t}: P_{p, 3}^{e x t}: P_{p, 4}^{e x t}: P_{p, 5}^{e x t} \\
& =F_{1}^{e x t} \cdot \delta: F_{2}^{e x t} \cdot \delta: F_{3}^{e x t} \cdot \delta: F_{4}^{e x t} \cdot \delta: F_{5}^{e x t} \cdot \delta \\
& =F_{1}^{e x t}: F_{2}^{e x t}: F_{3}^{e x t}: F_{4}^{e x t}: F_{5}^{e x t}
\end{aligned}
$$

For kinetic energy of five-fingers:

$$
\begin{aligned}
& P_{k, 1}^{e x t}: P_{k, 2}^{e x t}: P_{k, 3}^{e x t}: P_{k, 4}^{e x t}: P_{k, 5}^{e x t} \\
& =\frac{1}{2} m_{1} v_{1}^{2}: \frac{1}{2} m_{2} v_{2}^{2}: \frac{1}{2} m_{3} v_{3}^{2}: \frac{1}{2} m_{4} v_{4}^{2}: \frac{1}{2} m_{5} v_{5}^{2} \\
& =m_{1} v_{1}^{2}: m_{2} v_{2}^{2}: m_{3} v_{3}^{2}: m_{4} v_{4}^{2}: m_{5} v_{5}^{2}
\end{aligned}
$$

Where the subscript expresses the finger. In addition, for a certain hand, $m_{1}: m_{2}: m_{3}: m_{4}: m_{5}$ is constant. Thus, to solve for allocation of total energy, the formula is as follows.

$$
\begin{aligned}
& P_{1}^{S}: P_{2}^{S}: P_{3}^{S}: P_{4}^{S}: P_{5}^{S} \\
& \propto F_{1}^{e x t}: F_{2}^{e x t}: F_{3}^{e x t}: F_{4}^{e x t}: F_{5}^{e x t} \\
& \propto v_{1}^{2}: v_{2}^{2}: v_{3}^{2}: v_{4}^{2}: v_{5}^{2}
\end{aligned}
$$

The formula is applied to Fig. 4, and the proportional constant is selected by the visualization effect. The contribution lies in providing the method to select representative energy modes (see Movie S6). Additional information regarding the devices can be found in the Supplementary Materials and movies.

\section{b. Data collection}

The participants were individually seated in a comfortable chair in front of a table and were asked to place left hands on the table and watch the LCD monitor. A spongy cushion supported the arm with EMG electrodes, and the fingers were fixed in the fixed device. All subjects participated in the data collection and were included in off-line analyses. Besides, the EMG signals from subject 10 are contaminated with noise due to electrode shifts [28] and one lift-off electrode [29] intentionally. The subject was tasked with moving virtual fingers simultaneously to achieve the target gestures (contain ten energy modes in Movie S6). The task consisted of the target movements of five-fingers, single-finger, twofingers, and three-fingers flexion and extension simultaneously.

\subsubsection{Online experiment stages.}

The interface mainly originates from the adaptive mechanism of fingers in life, so the experimental design to prove the validity should regard activities of daily living (ADLs) as a reference. The verification is based on three aspects: (1) decoding untrained configurations, (2) decoding the amount of single-finger energy, and (3) real-time control of single-finger energy.

After training, some energy models using artificial neural network (ANN) learning method was obtained (see Data analysis part). We performed the following experiments using these models.

a.Experiment 1: the expression of unlearned continuous hand motions

\begin{tabular}{cc}
\hline Attribute & Condition \\
\hline Prepared models & $\begin{array}{c}\text { (1) Models with non-ICA } \\
\text { (2) Models with ICA }\end{array}$ \\
\hline hand & $\begin{array}{c}\text { (1) Trained hand } \\
\text { (2) Untrained hand }\end{array}$ \\
\hline Drive system & Virtual hand \\
\hline $\begin{array}{c}\text { With the evolution of limb in humans, hands have } \\
\text { developed into a highly sophisticated system used for }\end{array}$
\end{tabular}


manipulative activities - tool use, preparing, and eating food $[24,31]$. Today, as a result of cultural pressure, the complexity of the human hand motions has increased tremendously. Some tasks require different hand motions, such as turning a door handle or grabbing a car key. Other tasks require a more differentiated role for each finger, such as sewing, clicking the keyboard, playing musical instruments.

Some myoelectric interface implements the classification of many hand motions depending on large training datasets of target motions, resulting in an increased burden on users $[11,18]$. We, therefore, sought to implement the expression of multiple unlearned hand motions by our energy-based interface only using a few energy modes.

To assess the benefit, we asked participants to perform these hand tasks in figure $5 \mathrm{e}$ as faster as possible from figure $5 \mathrm{e} 1$ to figure $5 \mathrm{e} 13$, continuously rather than individually. Referring to the literature [32] and considering the adaptive adjustment in ADLs, we examined two outcome measures: completion rate and completion time. Trial failure was defined as the participant voluntarily gave up the trail, analogous to give up manual tasks that use the prosthetic hand in ADLs.

Six subjects (subject $5,6,7,8,9,10$ ) participated in this experiment. For this experiment, a total of 4 different experimental conditions were examined (Table S1 and Fig. 5). Tests under each condition were performed five times. We first test the trained hand and later test the untrained hand, and alternately test the algorithm with ICA and without for each hand. Additionally, for the trained hand test, we did not re-position the electrodes relative to the training phase, and for the untrained hand test, we re-positioned the electrodes. Also, for normalized EMG reducing individual differences, at the beginning of the experiment, we asked participants to flex and extend their hands and fingers to try maximum contractions for the muscles in the forearm.

b. Experiment 2: the amount of single finger energy

\begin{tabular}{cc}
\hline Attribute & Condition \\
\hline Prepared models & Models with ICA \\
\hline hand & $\begin{array}{c}\text { (1) Trained hand } \\
\text { (2) Untrained hand }\end{array}$ \\
\hline Drive system & Bionic hand \\
\hline
\end{tabular}

When manipulating objects, our native hands are good at exerting just exactly enough fingertip force on it [33]. For example, while the object is light and "fragile", such as a grape, our hands manipulate a "gentle" enough pinch not to cause any damage; while the object is heavy and slippery, such as a hammer, our hands can exert just enough pressure on an object to avoid slipping free from a stable grasp [34].

Some tests of manual dexterity do few benefits from force sensitivity - no penalty is incurred for exerting too much force on an object, such as the Box and Blocks Test and Action Research Arm Test. However, many tasks in activities of daily living (ADLs) are highly dependent on force sensitivity. In our test, we, therefore, designed the experiment in which prick suspended balloon with a needle. The balloon is "fragile" and "breaks up" if exerted finger energy too much, while the balloon is "suspended" and "slips away" if applied finger energy is too little.

To test whether the energy-based interface distinguishes the amount of finger energy, we had the participant repeatedly perform these selected hand motions by controlling a bionic hand whose fingertips were fitted with steel needles on the premise of ensuring breaking/nonbreaking the balloon (Fig. 6e and Movie S4).

Five subjects (subject 5,6,7,8,9) participated in this experiment. For this experiment, a total of 4 different experimental conditions were examined (Table S2 and Fig. $6)$. Tests under each condition were performed ten times. We first test the trained hand and later test the untrained hand, and alternately test the experiment with breaking or nonbreaking the balloon. When the participant was asked to perform the selected hand motion without breaking the balloon, trial failure was defined as "breaking" the balloon or not accomplishing the selected gesture. When the participant was asked to perform the selected hand motion with breaking the balloon, trial failure was defined as "non-breaking" the balloon or not accomplishing the selected gesture.

Additionally, the perimeter of the balloon is was about 66 $\mathrm{cm}$, and the length of the hanging rope is about $7 \mathrm{~cm}$. The experimental balloons were counterbalanced to reduce quality effects. Each balloon is filled with 36 grams of plasticine (Fig. S5).

\section{b. Experiment 3: the control of single finger energy in} real-time

\begin{tabular}{cc}
\hline Attribute & Condition \\
\hline Prepared models & Models with ICA \\
\hline hand & (1) Trained hand \\
& (2) Untrained hand \\
\hline
\end{tabular}

Drive system

Bionic hand

Our native hands were exquisitely proficient at flexing the finger a just enough position and perform manual tasks precisely - e.g., combing hair or applying lipstick, depending on controlling finger energy in real-time [24]. To display noticeable results when exerting too much energy, we developed a closed-loop task punching a hole in the plasticine $(\sim 1 \mathrm{~mm}$ thickness) attached to the fixed balloon with steel needles (Fig. 7e and Movie S5). Concretely, we had the participant repeatedly punch a hole in the plasticine ( $\sim 1 \mathrm{~mm}$ thickness) attached to the fixed balloon by using the index, middle and ring fingers, while not breaking the balloon.

Five subjects (Subject 5,6,7,8,9) participated in this experiment. For this experiment, both hands and selected fingers were examined (Table S3 and Fig. 7). Tests under 

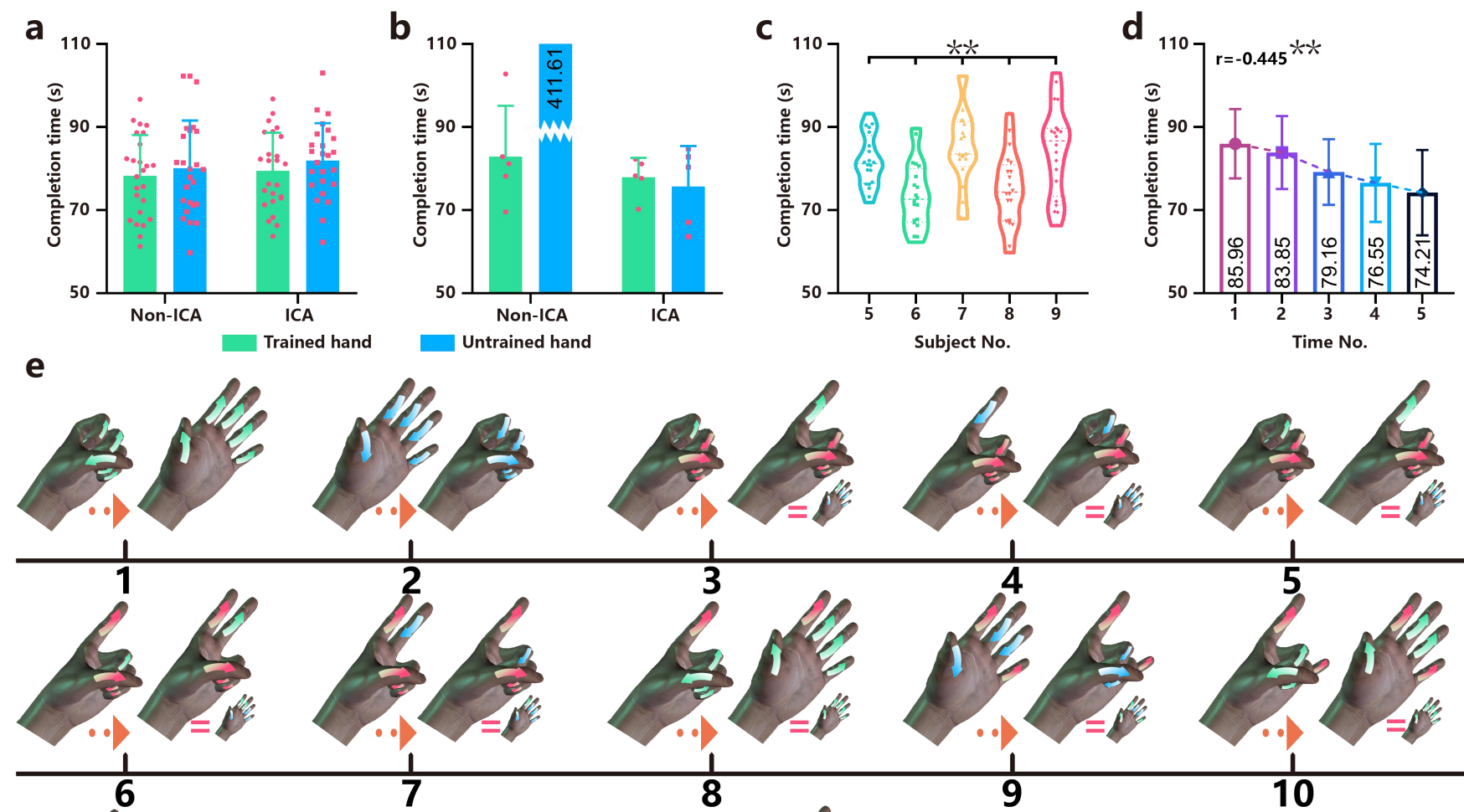

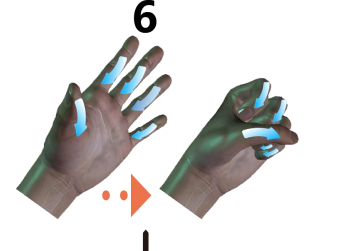

11

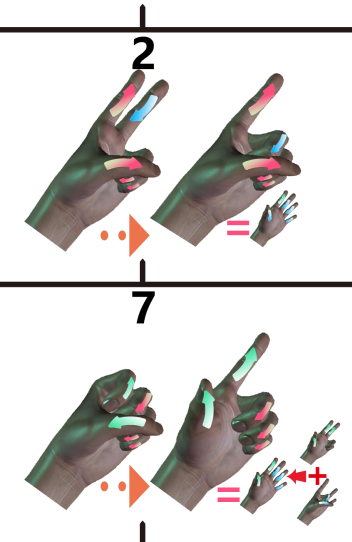

12
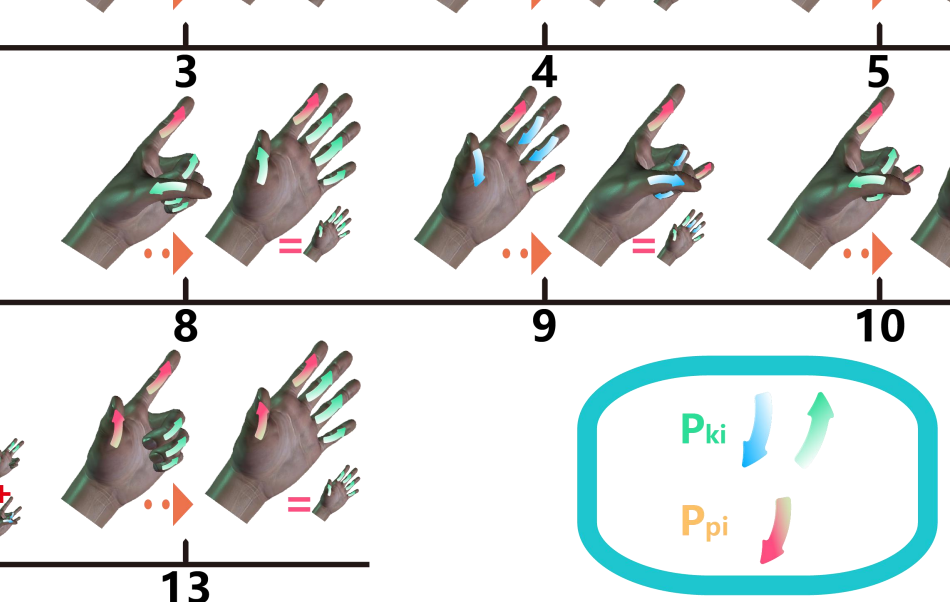

13

Fig. 5. Energy-based interface implements the expression of unlearned hand motions. To test the expression of multiple hand motions based on fundamental energy mode, we had the participant repeatedly sequential perform these selected hand motions as faster as possible (repeated 5 times under each condition). (a) The completion results of normal participants (subject 5-9). (b) The completion result of the participants whose EMG signals were contaminated with noise in training stage (subject 10). (c) Differences among participants in completion time (subject 5-9). (d) Faster with the number of operations. (e) Selected hand motions based on fundamental energy mode. [Note that, one continuous energy mode can adaptively extend to unlearned multiple motions or forces according to the task itself (e.g., mechanical coupling of task and physiological coupling of five-fingers) - e.g., the same energy model of e3 and e7; The unlearned energy mode can be expressed by fundamental energy mode-e.g., e12](In terms of motions, unlearned motions includes e3, e4, e5, e6, e7, e12, and e13). $P_{k i}$ expresses the kinetic energy of the $i$-th finger, while $P_{p i}$ expresses the potential energy of the $i-$ th finger. $* \mathrm{p}<0.05$, $* * \mathrm{p}<0.01$. Data show means \pm SD.

each condition were performed ten times. Success failure was defined as "punching" a hole with the selected finger under the unbroken balloon in 30 seconds (Fig. 7e and movie S5).

\subsection{Data analysis}

The formal scheme for the analysis of the EMG signals for the model: preprocessing steps, feature extraction, and the learning of models for EMG features and five-fingers energy features.

\subsubsection{Data processing.}

This preprocessing step included two further preprocessing steps. During EMG recording, 16-channel EMG data has been band-pass filtered from $10 \mathrm{~Hz}$ to $450 \mathrm{~Hz}$ and notch filtered of $50 \mathrm{~Hz}$ to remove movement artifacts, high-frequency noise, and power line noise and its harmonics [35]. Firstly, by considering the clinical relevance of using single-differential EMG, the EMG data of 16-channels were further processed to produce 8 bipolar channels by subtracting each pair of adjacent channels along the muscle fibers as they are more tolerant of noise than monopolar ones [36]. Additionally, after the first step, ICA can be selected. In order to reduce the output variable dimensions, the finger power of one finger was calculated as the pressure of finger pulp minus the pressure of the finger dorsum. Finally, the power data of 10-channels were processed to 5-channels wherein the signs of the power represented the flexure and extension of fingers, and the absolute values of the power represented the magnitude of power. Secondly, a $200 \mathrm{~ms}$ sliding window with a $50 \mathrm{~ms}$ overlap was used to downsampled to $6.67 \mathrm{~Hz}$ due to the difference in the sampling frequency between the EMG data and power data. The EMG data in the sliding window were prepared for feature extraction. The power data was filtered using a moving average window to improve movement smoothness towards online control. (more information in Supplementary 
Materials)

\subsubsection{Feature extraction.}

The fundamental purpose of feature extraction is to emphasize the critical information in the recording signal while rejecting noise and irrelevant data. We chose two groups.

Over the past two decades, some EMG features have been widely used in research and clinical practice. In this study, six time-domain features and two frequency-domain features typically used for myoelectric interfaces [35, 36] were extracted from each EMG channel in the $200 \mathrm{~ms}$ sliding windows producing a set of $64 \mathrm{EMG}$ features ( 8 features $\times 8$ channels). [E-T: Mean of absolute values, Variance, Waveform Length, Root-mean-square value, Willison Amplitude (WAMP), Zero crossing (ZC), Median Frequency (MDF), and Mean Frequency (MNF)]

Additionally, the EMG amplitude is a simple and useful feature, as evidenced by commercial prostheses [10]. To further improve the robustness to noise distinguishable by frequency band, we also extract the frequency-domain power (F-P) as features with a sample short-time Fourier transform, similar to amplitude in the different frequency band (Fig. S4), which produces a set of 88 EMG features (11 features $\times 8$ channels).

\subsubsection{Learning methods.}

As two examples of learning methods, we explore two learning methods. Firstly, a multi-layer feedforward ANN was used to learn a mapping between the EMG signals and the five-fingers energy. The functional relationship predicted by the ANN can be written as:

$$
P^{\text {pre }}(t)=N N(e(t), w)
$$

Where $P^{\text {pre }}(t) \in \mathfrak{R}^{5 \times 1}$ are the predicted five-fingers power, $e(t) \in \mathfrak{R}^{64 \times 1}(\mathrm{E}-\mathrm{T})$ or $e(t) \in \mathfrak{R}^{88 \times 1}(\mathrm{~F}-\mathrm{P})$ represent the EMG features, $w$ are the weight parameters which represent the links between the nodes or neurons. The network is made up of an input layer, a hidden layer with a tanh activation function (the number of neurons: 10), and a single linear output layer. The training algorithm was Levenberg-Marquardt back-propagation. Secondly, the excellent performance of the support vector machine (SVM) applied to regression problems is known. SVM regression is statistical learning machines [37] that build an approximated map between samples drawn from an input space (under the standard i.i.d. sampling hypothesis) and a set of real value. As is standard, we use the radial basis function for regression.

\subsubsection{Operational experiments.}

For all operational experiments, as an example, we used a $200 \mathrm{~ms}$ sliding window to extract F-P features and the ANN learning method to predict the energy of five-fingers online, and the online instruction update rate was kept at $200 \mathrm{~Hz}$ (5ms interval).

\section{Results}

\subsection{Experiment 1: energy-based interface achieves the expression of unlearned continuous hand motions}

All normal participants completed tasks successfully (100 of 100 times from subject 5-9; binomial test, $p<0.0001$;

Fig. 5a and Movie S1-3).

The energy-based interface requires the sensor signals from one hand, such as in the case of unilateral amputees, in training stages [38]. To test the performance of the proposed interface in the untrained hand, we had the participant respectively perform the above tasks with the trained hand or the untrained hand. As might be expected, given the similarity of both hands of one man in neuromuscular patterns, participants implement these motions not significantly slower with the untrained hand than the trained hand through either ICA $(81.96 \pm 8.95 \mathrm{~s}$ versus $79.47 \pm 9.15 \mathrm{~s}$; paired $t$ test, $p=0.210$; Fig. $4 \mathrm{a}$ ) or non-ICA algorithms $(80.10 \pm 11.48 \mathrm{~s}$ versus $78.27 \pm 9.80 \mathrm{~s}$; paired $t$ test, $p=0.435$; Fig. 5a ). However, there were significant differences among participants (one-way ANOVA test, $p<0.01$; Fig. 5 c), and it becomes significantly faster with the number of operations (correlation analysis, $\mathrm{r}=-0.445, p<0.01$; Fig. $5 \mathrm{~d}$ ).

Additionally, the lack of robustness of myoelectric control approaches is one of the reasons for the limited transfer into clinical and commercial applications [39]. To test whether the bionic interface that includes the ICA matrix was resistant to the interference of noise, we had subject 10 , respectively, perform the above tasks with the algorithms of non-ICA or ICA. We found that subject 10 was able to perform these tasks with the untrained hand through ICA algorithms ( 5 of 5 times; binomial test, $p<0.0001$; Fig. 5b), and as likely fast as with the trained hand $(75.73 \pm 9.69 \mathrm{~s}$ versus $77.90 \pm 4.69 \mathrm{~s}$; paired $t$ test, $p=0.621$; Fig. 5b ). However, subject 10 might be failed to achieve these tasks with the untrained hand through non-ICA algorithms (success times: 1 of 5 times; binomial test, $p=0.375$; Fig. $5 b$ ). The ICA that mimics the muscle synergy of the neuromuscular system in the training stage increased the robustness of the myoelectric interface [Although participants implement these motions not significantly faster with ICA than without (untrained hand: $81.86 \pm 8.95 \mathrm{~s}$ versus $80.10 \pm 11.48 \mathrm{~s}$; paired $t$ test, $p=0.429$; Fig. 5a)].

\subsection{Experiment 2: energy-based interface controls the amount of single finger energy}

The participant was able to exert just enough finger energy significantly better than chance (breaking: 366 of 500 trials; binomial test, $p<0.0001$; non-breaking: 413 of 500 trials; binomial test, $p<0.0001$; Fig. 6a). Besides, there was no significant difference between untrained hand and trained hand in the control of the amount of finger energy (nonbreaking: $8.20 \pm 1.19$ times versus $8.32 \pm 1.11$ times; paired $t$ 

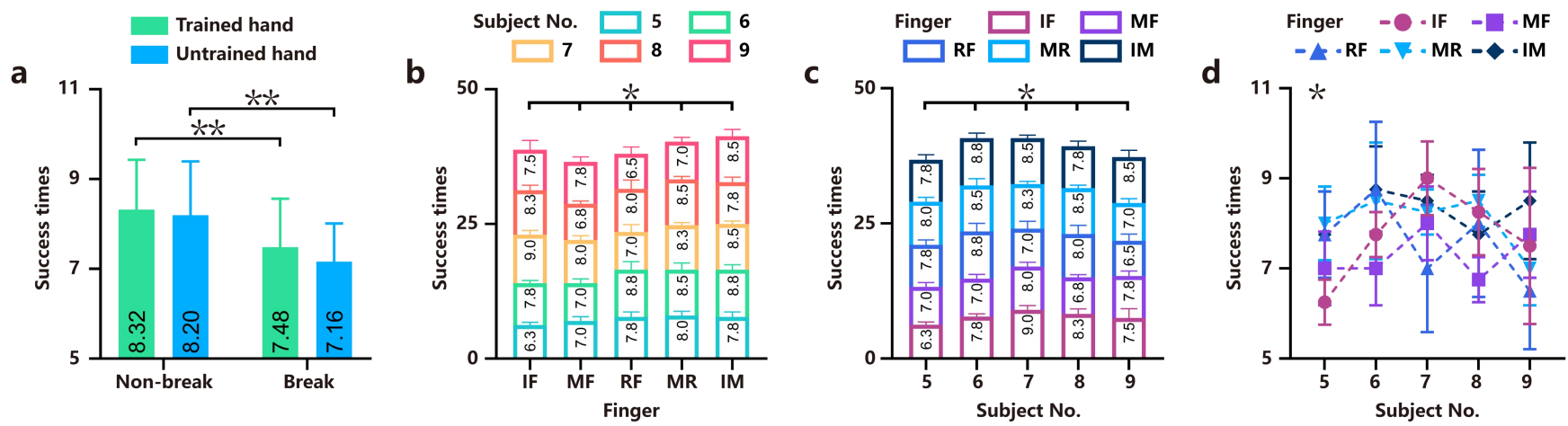

e
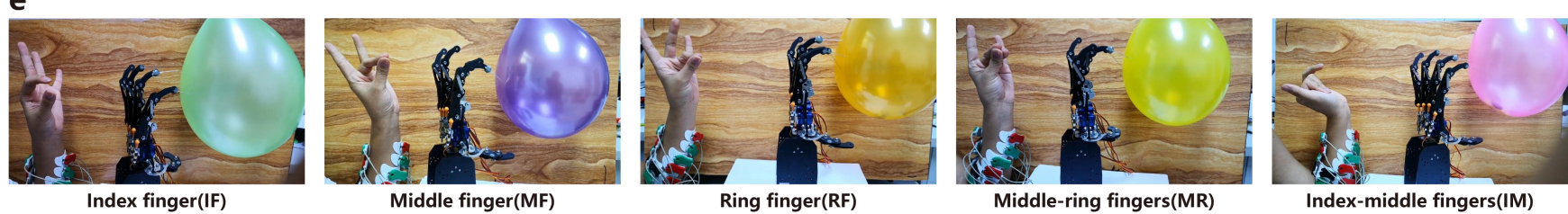

Fig. 6. Energy-based interface controls the amount of finger energy on the tasks pricking suspended balloons with a steel needle. To test whether the energybased interface distinguishes the amount of finger energy, we had the participant repeatedly perform these selected hand motions by controlling a bionic hand whose fingertips were fitted with steel needles, while ensuring breaking/non-breaking the balloon (repeated 10 times under each condition; subject 59). (a) Participants could exert just enough finger energy on a balloon. (b) Differences among fingers. (c) Differences among subjects. (d) Interaction effect between subjects and fingers. (E) Selected hand tasks according to ADLs. ${ }^{*} \mathrm{p}<0.05,{ }^{* *} \mathrm{p}<0.01$. Data show means \pm SD.

test, $p=0.671$; breaking: $7.16 \pm 0.85$ times versus $7.48 \pm 1.08$ times; paired $t$ test, $p=0.148$; Fig. 6a).

Contrary to expectation, it seemed not easy to prick suspended balloons with a needle relative to non-breaking counterpart (trained hand: $7.48 \pm 1.08$ times versus $8.32 \pm 1.11$ times; paired $t$ test, $p<0.01$; untrained hand: $7.16 \pm 0.85$ times versus $8.20 \pm 1.19$ times; paired $t$ test, $p<0.01$; Fig. 6a). One possibility, then, was the effect of balloons themselves. Thus, taking into consideration that a balloon is either breakable or hard to break, we explored the property of finger energy sensitivity by incorporating these two trials (break and non-break) for reducing or eliminating potential confound of balloons. We found that there might be significant differences across fingers and subjects at the control of the amount of finger energy (Two-way ANOVA test, fingers: $P<0.05$; Fig. $6 \mathrm{~b}$; subjects: $p<0.05$; Fig. $6 \mathrm{c}$ ), and different subjects might be adept in different fingers (Two-way ANOVA test, $p<0.05$; Fig. 6d).

\subsection{Experiment 3: energy-based interface controls single finger energy in real-time}

The participant punched a hole without breaking balloon at over $95 \%$ success rate $(97.67 \pm 5.04 \%$ versus $95 \%$; onesample $t$ test, $\underline{p}<0.01$; Fig. 7a). Furthermore, success rates with untrained hand and trained hand showed no significant difference in real-time, no matter which fingers were used (index finger: $100 \pm 0.00 \%$ versus $98 \pm 4.47 \%$; paired $t$ test, $p=0.374$; middle finger: $96 \pm 5.48 \%$ versus $98 \pm 4.47 \%$; paired $t$ test, $p=0.621$; ring finger: $96 \pm 8.94 \%$ versus $98 \pm 4.47 \%$; paired $t$ test, $p=0.704$; Fig. $7 \mathrm{a}$ ).

\subsection{Off-line analysis}

In the studies described above, the energy-based interface has been shown to confer functional benefits through three sets of operational experiments. Our purpose for this analysis was to explore the characteristics of the energy-based interface further and explain why the interface shows great functional benefits. Two performance indices were chosen to evaluate the accuracy of the estimation in each finger energy. Pearson's correlation coefficient (R) was calculated to assess the total variation between the estimated and actual energy, while the root-mean-square error (RMSE) to describe the total residual error.

\subsubsection{Energy-based interface achieves a continuous estimation of finger energy in real-time.}

Even an element of EMG datasets exists massive properties, the properties that similar in the group, but different among groups should be selected as features for a better model, and learning methods should be adopted to distinguish these groups through features as far as possible. To the extent, the fitting model is the classification model whose classes is infinite and continuous.

To assess which combination of features and learning methods could apply to the energy-based interface, a ten-fold cross-validation procedure was used to evaluate the overall statistical performance of both different features (E-T and FP) and learning methods (ANN and SVM). Figure 8 shows the continuous estimation results from 10 subjects for 10 test trials. The signs of value represent the flexion or extension of fingers, and the absolute values represent the amount of finger energy. As an example, the equivalent state of kinetic energy and potential energy is shown from the data of subject 4. Figure $\mathrm{S} 6$ shows the confusion matrix for the estimation of the finger energy of subject 4 . Although the confusion matrix 

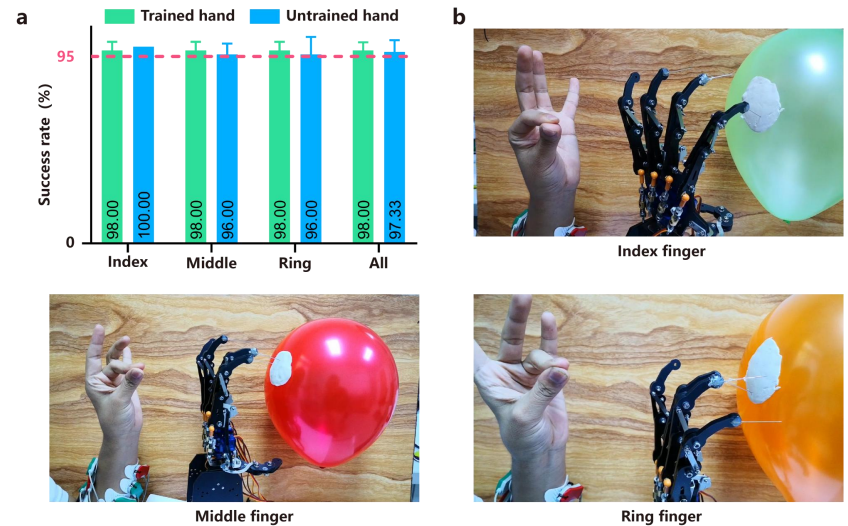

Fig. 7. Energy-based interface controls the single finger energy in real-time on the tasks punching a hole with a steel needle. To assess the degree to control the finger energy in real-time, we had the participant repeatedly punch a hole in the plasticine ( $\sim 1 \mathrm{~mm}$ thickness) attached to the fixed balloon by using single fingers, while not breaking the balloon (repeated 10 times under each condition; subject 5-9). (a) Participants could flex the finger a just enough position to punch a hole on the plasticine. (b) Example of tasks for the index, middle and ring fingers. Data show means $\pm \mathrm{SD}$.

shows some deviations, the user can correct the deviations in real-time, similar to the native hand $[40,41]$. Furthermore, we found ANN outperformed SVM, whether in the total variation $(0.699 \pm 0.124$ versus $0.653 \pm 0.125$; Three-way ANOVA test, $p<0.01$; Fig. S7C) or the total residual error $(0.209 \pm 0.040$ versus $0.223 \pm 0.041$; Three-way ANOVA test, $p<0.01$; Fig. S7F). Also, there are significant differences across fingers (R: Three-way ANOVA test, $p<0.01$; Fig. S7B. RMSE: Three-way ANOVA test, $p<0.01$; Fig. S7E). The lowly individuated fingers (ring and middle fingers) are likely performances better in the total variation [2].

Another way to assess the trait of the energy-based interface relative to classification-based is to characterize the accuracy of the estimation in a certain range of the amount of energy [13]. To test this extraordinary capability for single finger energy, we divided the amount of energy from zero to maximum voluntary energy (MVE) into 5 ranges (normalized energy; Fig. 9a and Fig. 9c). The among ranges difference was significant, whether in the total variation (One-way ANOVA test, $p<0.01$; Fig. 9a) or the total residual error [One-way ANOVA test, $p$ $<0.01$ (logarithm of RMSE); Fig. 9a]. Also, the total residual error increase with the improvement in energy (correlation analysis, $\mathrm{r}=0.961, p<0.01$; Fig. 9a ), similar to the native hand $[40,41]$. More interestingly, the distribution of relative energy (ratio of voluntary energy to MVE for the finger) is likely consistent with the usage frequency of the finger (Fig.9c). For instance, unconsciously exerting higher relative energy in the finger might signify more frequent use in ADLs. Furthermore, the model estimates the finger energy better for finger flexion than finger extension in total variation (the whole estimated energy relative to the whole actual energy rather than the statistics according to ten tests, $0.622 \pm 0.034$ versus $0.495 \pm 0.041$; paired $t$ test, $p$ $<0.01$; Fig. 9b), presumably reflecting better performances in
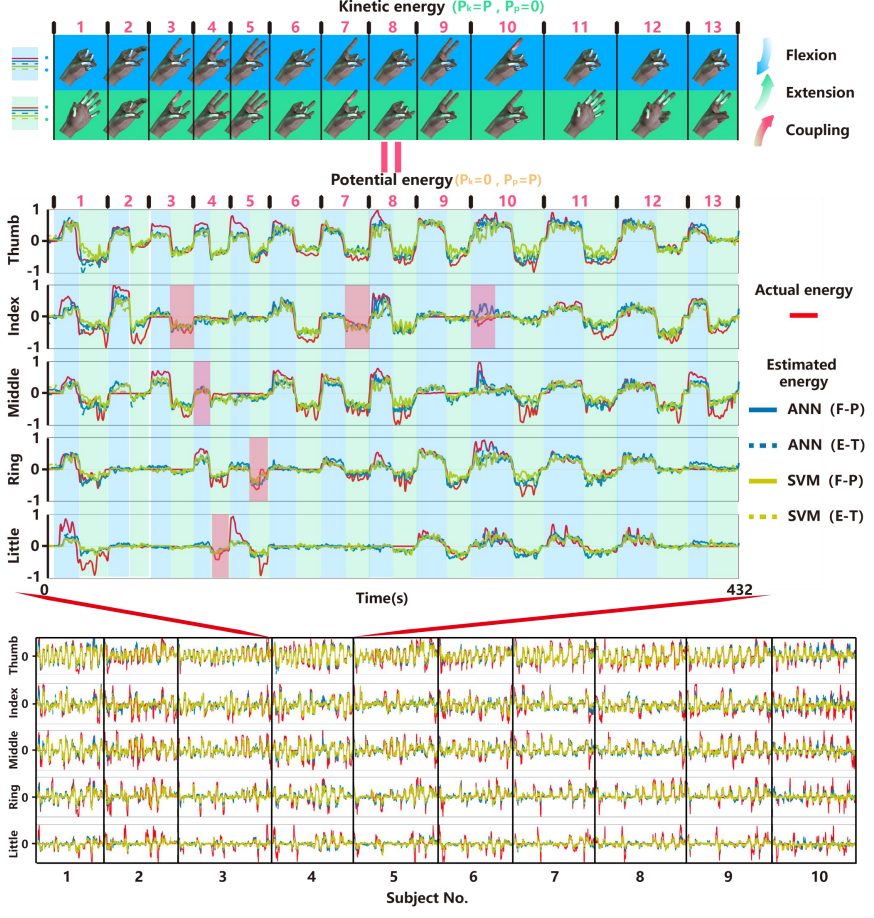

Fig. 8. Test data for ten subjects. Normalized five-fingers energy is shown in red solid lines, while the estimated results of the ANN and SVM method are shown in blue and green, respectively. The estimated energy of F-P features is shown in solid lines, and the energy of E-T features are shown in dotted lines. The signs of value represent the flexure or extension of fingers, and the absolute values represent the amount of finger energy. The data for subject 4 , as an example, show the equivalent state of kinetic energy and potential energy. Besides, the blue arrows and shade bars represents the flexure of fingers, the green arrows and shade bars represents the extension of fingers, while the pink arrows and shade bars represent the coupled motion of fingers. $P$ expresses the total energy of fingers, $P_{k}$ expresses the kinetic energy of fingers, and $P_{p}$ expresses the potential energy of fingers. Note that although some fingers remain stationary by overcoming the coupling, their muscle-energy modes are the same as in some hand motion.

grabbing and pinching.

\subsubsection{The generalization of across subjects is explored.}

Previous studies of muscle synergy for wrist demonstrated that humans have a similar anatomical structure and synergy $[42,43]$. To assess the degree to whether the energy-based interface applies to unlearned subjects, we used another tenfold cross-validation procedure whose testing datasets from one subject totally while training datasets from other subjects, relative to the previous test. Firstly, we found there are significant differences among subjects, whether in unlearned subjects (One-way ANOVA test, R: $p<0.01$; Fig. S8A; RMSE: $p<0.01$; Fig. S8F) or "learned" subject of the previous procedure (One-way ANOVA test; R: $p<0.01$; Fig. S8A. RMSE: $p<0.01$; Fig. S8F). Secondly, the "learned" subject outperformed the unlearned subject (paired $t$ test; $\mathrm{R}: 0.697 \pm 0.008$ versus $0.680 \pm 0.007, p<0.01$; Fig. S8D. RMSE: $0.215 \pm 0.041$ versus $0.230 \pm 0.047, p<0.01$; Fig. S8I), which presumably reflecting personalized anatomical structure [42]. Furthermore, as expected, when the training 

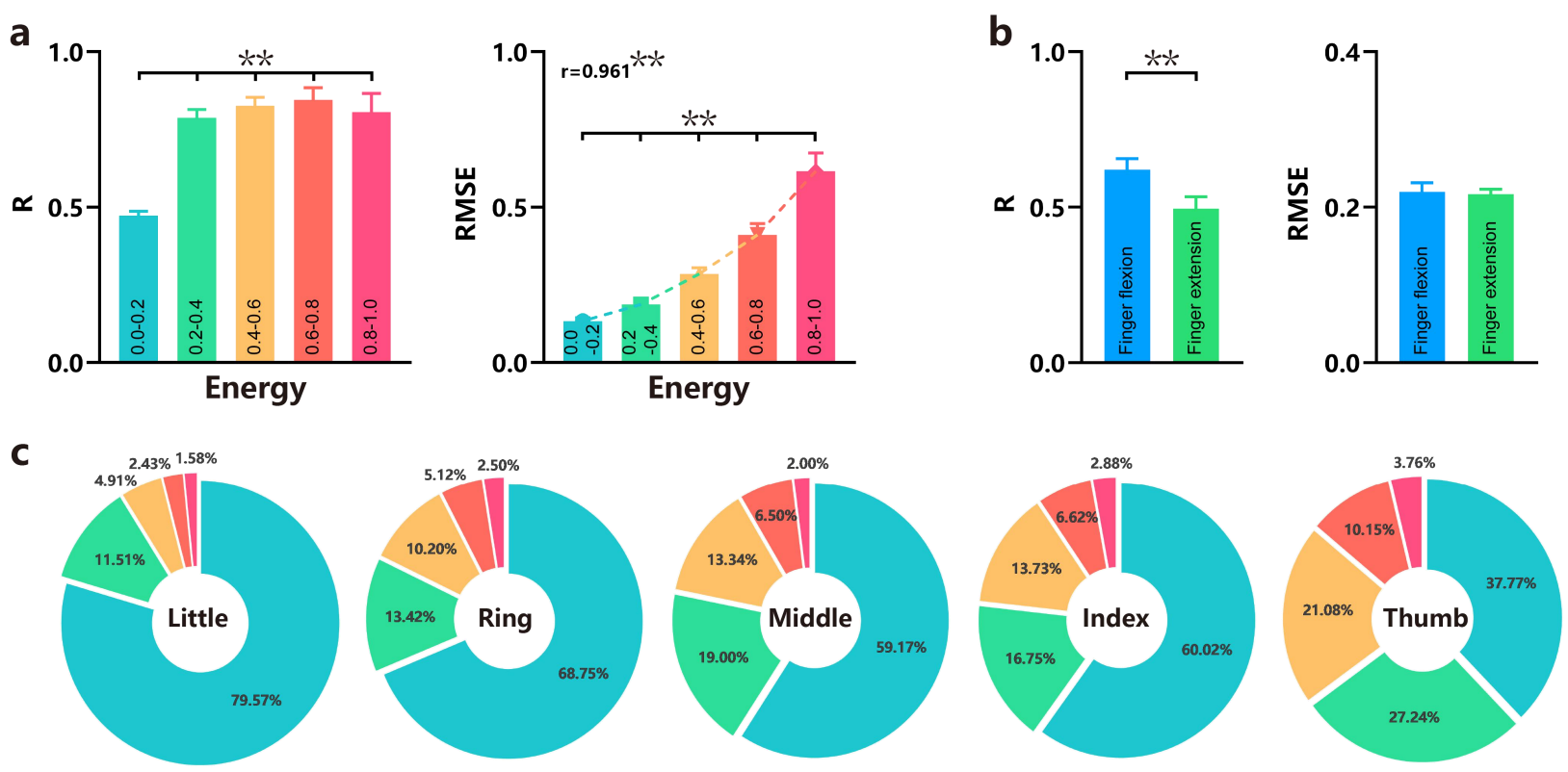

\begin{tabular}{l|l|l|l|l|l|} 
Energy & $0.0-0.2$ & $0.2-0.4$ & $0.4-0.6$ & $0.6-0.8$ & $0.8-1.0$
\end{tabular}

Fig. 9. The amount of five-fingers energy. To explore this extraordinary capability for a single finger in the amount of energy relative to the classification model, firstly, we divided the amount of energy from zero to maximum voluntary energy (MVE) into 5 ranges, and (a) explored the performance within each range; secondly, (c) we counted the distribution of these ranges for each finger, presumably consistent with the usage frequency of the finger in ADLs. Furthermore, for the performances in grabbing and pinching, (b) we also explored the accuracy according to the range of finger flexion and extension. ${ }^{* *}$ p $<$ 0.01 . Data show means \pm SD.

datasets did not contain the data of the testing subject, performance degradation in the subject contaminated with noise is more noticeable (Fig. S8C and Fig. S8H); we also observed a significant increase in the coefficient of variation (paired $t$ test; R:0.044 \pm 0.009 versus $0.092 \pm 0.021, p<0.01$; Fig. S8E. RMSE: $0.007 \pm 0.004$ versus $0.024 \pm 0.013, p<0.01$; Fig. S8J), highlighting the huge difference in performance across features and across learning methods for subject contaminated with noise.

\subsubsection{ICA mimicking muscle synergy improves the robustness of the energy-based interface.}

In the studies described above, when trained with the subject contaminated with noise, the model shows performance better with ICA than without for standard data (Fig. 5b), presumably relying on information separation capacity of ICA (Fig.4b) [44]. Furthermore, ICA has been applied to extract synergies from the muscles of frogs [26] and rats [27]. To further assess whether the ICA model trained with the standard data applies to the subject contaminated with noise, we rebuilt a model using the synergy matrix decomposed by standard data from subject 1 9. Also, the model evaluation was accomplished through 10fold cross-validation whose datasets divided by subjects. We found the model with ICA likely outperformed the previous model for the subject contaminated with noise (paired $t$ test; $\mathrm{R}: 0.286 \pm 0.148$ versus $0.257 \pm 0.171, p=0.057$; RMSE: $0.274 \pm 0.052$ versus $0.290 \pm 0.060, p<0.01$; Fig. S9). However, the model with ICA showed no advantage in normal conditions (Fig. S9B and Fig. S9D). Briefly, the major advantage of the ICA model lies in the capacity of reducing or eliminating the noise due to myoelectric prosthesis "aging" with time and use, such as failure electrodes and deformation, which increase the service life of the myoelectric prosthesis through recalibration (Fig.4b and Fig. 5b).

\section{Discussion and conclusion}

In the present study, we demonstrate that the dynamic energy model that decodes continuous hand actions with force information by training small amounts of sEMG data. This theory imitates the self-adapting mechanism to the coupling of the five-fingers in actual motion in the actual task. For example, to gesture "V" in Fig. 1, according to realtime feedback, humans usually unconsciously and adaptively control thumb to press ring and little fingers (translate the energy of these two fingers into potential energy). Thus, the experimental design to prove the validity should regard ADLs as a reference.

Experiment 1: First of all, performed with a certain task such as turning a door handle or grabbing a car key, the user must be able to enforce the correct grasping motions.

Considering continuous automatical adjusting of the human hand in ADLs, we have participants perform a series of uninterrupted actions. The results show that few continuous energy modes can adaptively combine into multiple motions or forces according to the task itself (e.g., mechanical coupling of the task and physiological coupling of five-fingers $[1,2]$ ), according to real-time feedback, similar to the human hand [40, 41]. Also, some unlearned 
energy mode can be expressed by learned energy mode. Besides, the correlation coefficients of off-line analysis are relatively low compared to previous researches in the wrist due to less training data and more DOFs [14-16]. However, because the method is designed by imitating mechanical coupling and physiological coupling of five-fingers in humans, regarding the whole manual task as an energy transfer, subjects can adaptively adjust the energy mode in real-time for the operational experiments, and results are excellent.

Experiment 2: Second, the amount of energy involved in the grasp must be controlled so that it is possible to grab, e.g., both a hammer without letting it slip and an egg without breaking it.

Use small energy to complete finger flexion to ensure that the balloon was not broken by the needle of a fingertip, or use abundant energy to complete finger flexion to ensure that the balloon was broken without moving away. Considering the influence of the quality of the balloon, we test both conditions for each balloon. Through overall consideration of both conditions, we prove that the presented model can achieve exerting just enough finger energy on "fragile" or "heavy" objects.

Experiment 3: Third, real-time control of single-finger energy is paramount so that it is possible to perform manual tasks precisely, e.g., applying lipstick.

Control finger energy in real-time to ensure punching a hole in the plasticine $(\sim 1 \mathrm{~mm}$ thickness $)$ attached to the fixed balloon without breaking the balloon. In actual operation, for our model, this task was easy to complete, and the success rate was over $95 \%$.

Overall, the study involved the main lines of research about the myoelectric interface. One line of research is to recognizing hand gestures based on pattern recognition. These related works focus on improving classification accuracy and the number of discriminated motions [11-13]. With adequately designed feature extraction and classifier, it is possible to achieve extremely high classification accuracies ( 90-95\%) on a large repertoire of hand motions (21 classes) [12]. In 2019, a combined hand-motions viewpoint emerged [13]. The literature [13] showed some unlearned combined motions could be expressed using only learned single motions. However, this is a combination of discrete motions. Here, inheriting previous work, we propose a different way to extend hand tasks using continuous energy modes. Some different hand movements can be divided into the same energy modes from the five-fingers level. In actual operation, the user can accomplish a mass of hand movements by adjusting a few continuous energy modes adaptively according to feedback. This means that, for later research about sEMG gesture recognition, one may consider tagging the training data by energy mode instead of gesture.

Another line of research is to estimate a proportional activation of each DOF. These related works mainly focus on the wrist or hand close and open $[15,39,43,45,46]$.
However, the estimation of forces and kinematics of single finger has rarely been investigated. Unlike the wrist, both mechanical coupling and neuromuscular control limit to finger independence $[1,2]$. For instance, active movement at one finger may lead to some movement at another finger. Previous approaches that estimate a proportional activation for each DOF might not apply to five-fingers unless training using vast amounts of data. Our work explored the fivefingers as as a whole and divided massive manual tasks into several specific energy modes in terms of energy, thus, this problem was overcome.

In terms of signal selection and processing, a myoelectric signal comprises two states: a transient state emanating from a burst of fibers, as a muscle goes from rest to a voluntary contraction level, and a steady-state emanating during a consistently maintained contraction in a muscle [7]. The latter component dwarfs the former in robustness [47], and the energy-based control used the latter one. Besides, as with previous studies [20-23], the present experiments explored ICA to extract muscle synergy. Compared to directly use the sEMG features as the input of ANN, the model with ICA has shown no significant advantage inaccuracy. The machine learning methods, such as ANNs, were able to learn the mapping relationship consistent with ICA. However, before learning the relationship by machine learning methods, ICA could reduce or eliminate the noise of failure electrodes and unstable connections via recalibration (removing the interference-independent components). Such capacity may prove more and more valuable with long-term use. With the advent of more and more prosthetic hands, it may become an effective means of increasing the service life of commercial prostheses.

The main limitation of the current study is that no amputee was recruited. However, in previous studies, a large number of amputees were similarly demonstrated to the non-disabled subjects in muscle activation $[15,43]$, and it is not likely that the motor learning ability of the amputees would be greatly affected by the limb deficiency. Besides, our operational experiments showed that the performance degradation for using for the untrained hand was not significant when the model was trained by the contralateral limb of the same person. Of course, we exclude the amputee in muscular atrophy or non-existent measurable muscles for the application scope of the proposed method. The present study emphasizes the concept of hand action division by energy model, and the user is not limited to the amputee, e. g., neurorehabilitation after stroke [48-50].

Amputees have expressed a desire for intuitive myoelectric control [51]. The adaptive property of the energy-based interface underlines the importance of the capacity of the user to interact with the machine and learn a new task in which the user is within the loop and can adapt to the control system. Before concluding, it is worth mentioning that this study showed that, for later research about sEMG gesture recognition, one might consider tagging 
the training data by energy mode instead of gesture to reduce training complexity.

\section{References}

[1] C.E. Lang, M.H. Schieber, Human finger independence: Limitations due to passive mechanical coupling versus active neuromuscular control, J. Neurophysiol. 92(5) (2004) 28022810 .

[2] C. Hager-Ross, M.H. Schieber, Quantifying the independence of human finger movements: Comparisons of digits, hands, and movement frequencies, J. Neurosci. 20(22) (2000) 8542-8550.

[3] S. Raspopovic, M. Capogrosso, F.M. Petrini, M. Bonizzato, J. Rigosa, G. Di Pino, J. Carpaneto, M. Controzzi, T. Boretius, E. Fernandez, G. Granata, C.M. Oddo, L. Citi, A.L. Ciancio, C. Cipriani, M.C. Carrozza, W. Jensen, E. Guglielmelli, T. Stieglitz, P.M. Rossini, S. Micera, Restoring Natural Sensory Feedback in Real-Time Bidirectional Hand Prostheses, Sci. Transl. Med. 6(222) (2014) 10.

[4] B. Peerdeman, D. Boere, H. Witteveen, R.H. in't Veld, H. Hermens, S. Stramigioli, H. Rietman, P. Veltink, S. Misra, Myoelectric forearm prostheses: State of the art from a usercentered perspective, Journal of Rehabilitation Research and Development 48(6) (2011) 719-737.

[5] D. Farina, N. Jiang, H. Rehbaum, A. Holobar, B. Graimann, H. Dietl, O.C. Aszmann, The Extraction of Neural Information from the Surface EMG for the Control of UpperLimb Prostheses: Emerging Avenues and Challenges, IEEE Trans. Neural Syst. Rehabil. Eng. 22(4) (2014) 797-809.

[6] D.S. Childress, CLOSED-LOOP CONTROL IN PROSTHETIC SYSTEMS - HISTORICAL-PERSPECTIVE, Annals of Biomedical Engineering 8(4-6) (1980) 293-303.

[7] M.A. Oskoei, H.S. Hu, Myoelectric control systems-A survey, Biomed. Signal Process. Control 2(4) (2007) 275294.

[8] L. McLean, R.N. Scott, The Early History of Myoelectric Control of Prosthetic Limbs (1945-1970), in: A. Muzumdar (Ed.), Powered Upper Limb Prostheses: Control, Implementation and Clinical Application, Springer Berlin Heidelberg, Berlin, Heidelberg, 2004, pp. 1-15.

[9] P. Parker, K. Englehart, B. Hudgins, Myoelectric signal processing for control of powered limb prostheses, Journal of Electromyography and Kinesiology 16(6) (2006) 541-548.

[10] Ottobock, Bebionic technical manual. www.ottobockus.com/media/local-media/prosthetics/upperlimb/files/14112 bebionic user guide lo.pdf.

[11] M. Atzori, M. Cognolato, H. Muller, Deep Learning with Convolutional Neural Networks Applied to Electromyography Data: A Resource for the Classification of Movements for Prosthetic Hands, Front. Neurorobotics 10 (2016) 10.

[12] Y.J. Geng, Y. Ouyang, O.W. Samuel, S. Chen, X. Lu, C. Lin, G. Li, A Robust Sparse Representation Based Pattern Recognition Approach for Myoelectric Control, IEEE Access 6 (2018) 38326-38335.

[13] A. Furui, S. Eto, K. Nakagaki, K. Shimada, G. Nakamura, A. Masuda, T. Chin, T. Tsuji, A myoelectric prosthetic hand with muscle synergy-based motion determination and impedance model-based biomimetic control, Sci. Robot. 4(31) (2019) eaaw6339.

[14] N. Jiang, J.L.G. Vest-Nielsen, S. Muceli, D. Farina, EMG-based simultaneous and proportional estimation of wrist/hand kinematics in uni-lateral trans-radial amputees, J. NeuroEng. Rehabil. 9 (2012) 11.

[15] J.M. Hahne, F. Biessmann, N. Jiang, H. Rehbaum, D. Farina, F.C. Meinecke, K.R. Muller, L.C. Parra, Linear and Nonlinear Regression Techniques for Simultaneous and Proportional Myoelectric Control, IEEE Trans. Neural Syst. Rehabil. Eng. 22(2) (2014) 269-279.

[16] S. Muceli, D. Farina, Simultaneous and Proportional Estimation of Hand Kinematics From EMG During Mirrored Movements at Multiple Degrees-of-Freedom, IEEE Trans. Neural Syst. Rehabil. Eng. 20(3) (2012) 371-378.

[17] K.Z. Zhuang, N. Sommer, V. Mendez, S. Aryan, E. Formento, E. D’Anna, F. Artoni, F. Petrini, G. Granata, G. Cannaviello, W. Raffoul, A. Billard, S. Micera, Shared human-robot proportional control of a dexterous myoelectric prosthesis, Nature Machine Intelligence 1(9) (2019) 400-411. [18] R.N. Khushaba, S. Kodagoda, Ieee, Electromyogram (EMG) Feature Reduction Using Mutual Components Analysis for Multifunction Prosthetic Fingers Control, 2012 12th International Conference on Control, Automation, Robotics \& Vision, Ieee, New York, 2012, pp. 1534-1539.

[19] N. Jiang, K.B. Englehart, P.A. Parker, Extracting Simultaneous and Proportional Neural Control Information for Multiple-DOF Prostheses From the Surface Electromyographic Signal, IEEE Trans. Biomed. Eng. 56(4) (2009) 1070-1080.

[20] M.C. Tresch, P. Saltiel, E. Bizzi, The construction of movement by the spinal cord, Nature Neuroscience 2(2) (1999) 162-167.

[21] L.H. Ting, J.M. Macpherson, A limited set of muscle synergies for force control during a postural task, J. Neurophysiol. 93(1) (2005) 609-613.

[22] S.A. Overduin, A. d'Avella, J. Roh, E. Bizzi, Modulation of muscle synergy recruitment in primate grasping, J. Neurosci. 28(4) (2008) 880-892.

[23] A. d'Avella, A. Portone, L. Fernandez, F. Lacquaniti, Control of fast-reaching movements by muscle synergy combinations, J. Neurosci. 26(30) (2006) 7791-7810.

[24] J.R. Napier, THE PREHENSILE MOVEMENTS OF THE HUMAN HAND, J. Bone Joint Surg.-Br. Vol. 38(4) (1956) 902-913.

[25] M.R. Cutkosky, ON GRASP CHOICE, GRASP MODELS, AND THE DESIGN OF HANDS FOR MANUFACTURING TASKS, IEEE Trans. Robot. Autom. 5(3) (1989) 269-279.

[26] C.B. Hart, S.F. Giszter, Modular premotor drives and unit bursts as primitives for frog motor behaviors, J. Neurosci. 24(22) (2004) 5269-5282.

[27] W.J. Kargo, D.A. Nitz, Early skill learning is expressed through selection and tuning of cortically represented muscle synergies, J. Neurosci. 23(35) (2003) 11255-11269.

[28] A.J. Young, L.J. Hargrove, T.A. Kuiken, The Effects of Electrode Size and Orientation on the Sensitivity of 
Myoelectric Pattern Recognition Systems to Electrode Shift, IEEE Trans. Biomed. Eng. 58(9) (2011) 2537-2544.

[29] S. Amsuess, P. Goebel, B. Graimann, D. Farina, A Multi-Class Proportional Myocontrol Algorithm for Upper Limb Prosthesis Control: Validation in Real-Life Scenarios on Amputees, IEEE Trans. Neural Syst. Rehabil. Eng. 23(5) (2015) 827-836.

[30] CHAPTER 2 - Anatomy and Kinesiology of the Upper Extremity, in: E.E. Fess, K.S. Gettle, C.A. Philips, J.R. Janson (Eds.), Hand and Upper Extremity Splinting (Third Edition), Mosby, Saint Louis, 2005, pp. 47-85.

[31] F. Galis, J.J.M. van Alphen, J.A.J. Metz, Why five fingers? Evolutionary constraints on digit numbers, Trends Ecol. Evol. 16(11) (2001) 637-646.

[32] A. Ameri, E.N. Kamavuako, E.J. Scheme, K.B. Englehart, P.A. Parker, Support Vector Regression for Improved Real-Time, Simultaneous Myoelectric Control, IEEE Trans. Neural Syst. Rehabil. Eng. 22(6) (2014) 11981209.

[33] E.D. Engeberg, S. Meek, Improved grasp force sensitivity for prosthetic hands through force-derivative feedback, IEEE Trans. Biomed. Eng. 55(2) (2008) 817-821.

[34] M. Domalain, L. Vigouroux, F. Danion, V. Sevrez, E. Berton, Effect of object width on precision grip force and finger posture, Ergonomics 51(9) (2008) 1441-1453.

[35] A. Sarasola-Sanz, N. Irastorza-Landa, E. Lopez-Larraz, F. Shiman, M. Spuler, N. Birbaumer, A. Ramos-Murguialday, Design and effectiveness evaluation of mirror myoelectric interfaces: a novel method to restore movement in hemiplegic patients, Sci Rep 8 (2018) 13.

[36] M. Hakonen, H. Piitulainen, A. Visala, Current state of digital signal processing in myoelectric interfaces and related applications, Biomed. Signal Process. Control 18 (2015) 334-359.

[37] C.C. Chang, C.J. Lin, LIBSVM: A Library for Support Vector Machines, Acm Transactions on Intelligent Systems and Technology 2(3) (2011) 27.

[38] J.L.G. Nielsen, S. Holmgaard, N. Jiang, K.B. Englehart, D. Farina, P.A. Parker, Simultaneous and Proportional Force Estimation for Multifunction Myoelectric Prostheses Using Mirrored Bilateral Training, IEEE Trans. Biomed. Eng. 58(3) (2011) 681-688.

[39] J.M. Hahne, M.A. Schweisfurth, M. Koppe, D. Farina, Simultaneous control of multiple functions of bionic hand prostheses: Performance and robustness in end users, Sci. Robot. 3(19) (2018) 9.

[40] K.J. Cole, D.L. Rotella, Old age impairs the use of arbitrary visual cues for predictive control of fingertip forces during grasp, Exp. Brain Res. 143(1) (2002) 35-41.

[41] C. Voelcker-Rehage, A.J. Stronge, J.L. Alberts, Agerelated differences in working memory and force control under dual-task conditions, Aging Neuropsychol. Cogn. 13(3-4) (2006) 366-384.

[42] C. Choi, J. Kim, Synergy matrices to estimate fluid wrist movements by surface electromyography, Medical Engineering \& Physics 33(8) (2011) 916-923.

[43] N. Jiang, H. Rehbaum, I. Vujaklija, B. Graimann, D. Farina, Intuitive, Online, Simultaneous, and Proportional
Myoelectric Control Over Two Degrees-of-Freedom in Upper Limb Amputees, IEEE Trans. Neural Syst. Rehabil. Eng. 22(3) (2014) 501-510.

[44] A. Hyvarinen, E. Oja, Independent component analysis: algorithms and applications, Neural Networks 13(4-5) (2000) 411-430.

[45] N. Jiang, I. Vujaklija, H. Rehbaum, B. Graimann, D. Farina, Is Accurate Mapping of EMG Signals on Kinematics Needed for Precise Online Myoelectric Control?, IEEE Trans. Neural Syst. Rehabil. Eng. 22(3) (2014) 549-558.

[46] E.N. Kamavuako, K.B. Englehart, W. Jensen, D. Farina, Simultaneous and Proportional Force Estimation in Multiple Degrees of Freedom From Intramuscular EMG, IEEE Trans. Biomed. Eng. 59(7) (2012) 1804-1807.

[47] K. Englehart, B. Hudgins, P.A. Parker, A wavelet-based continuous classification scheme for multifunction myoelectric control, IEEE Trans. Biomed. Eng. 48(3) (2001) 302-311.

[48] Z.Y. Lu, K.Y. Tong, X. Zhang, S. Li, P. Zhou, Myoelectric Pattern Recognition for Controlling a Robotic Hand: A Feasibility Study in Stroke, IEEE Trans. Biomed. Eng. 66(2) (2019) 365-372.

[49] Y. Zhou, Y.F. Fang, K. Gui, K.R. Li, D.G. Zhang, H.H. Liu, sEMG Bias-Driven Functional Electrical Stimulation System for Upper-Limb Stroke Rehabilitation, IEEE Sens. J. 18(16) (2018) 6812-6821.

[50] D. Leonardis, M. Barsotti, C. Loconsole, M. Solazzi, M. Troncossi, C. Mazzotti, V.P. Castelli, C. Procopio, G. Lamola, C. Chisari, M. Bergamasco, A. Frisoli, An EMGControlled Robotic Hand Exoskeleton for Bilateral Rehabilitation, IEEE Trans. Haptics 8(2) (2015) 140-151.

[51] N. Jiang, S. Dosen, K.R. Muller, D. Farina, Myoelectric Control of Artificial Limbs-Is There a Need to Change Focus?, IEEE Signal Process. Mag. 29(5) (2012) 147-150. 